\begin{abstract}
Aus dem Institut für Angewandte Agrarokologie, Rostock', dem Forschungsinstitut für die Biologie landwirtschaflicher Nutztiere, Dummerstorf ${ }^{2}$ und dem Landesveterinăr- und Lebensmitteluntersuchungsamt Rostock ${ }^{3}$

HEINZ FALKENBERG', GERDA KUHN ${ }^{2}$, MARGITTA HARTUNG ${ }^{2}$, MARTINA LANGHAMMER ${ }^{2}$ und CAROLA WOLF ${ }^{3}$

\section{Verlauf von biochemischen Kennwerten im Blut von Schweinen mit unterschiedlicher Fettansatzleistung}

\begin{abstract}
Summary
Title of the paper: Level of the metabolic substances in blood in relation to the development of pigs with different capacity for lipid deposition

28 German Saddle Back pigs (DS) and 30 German Landrace pigs (DL) were used to determine the concentrations of 16 substrates and enzymes in the blood plasma during the growing-finishing period. Blood samples were taken from vena cava cranialis all two weeks 24 hours after Myostress injection. For the characterization of breed differences the results were represented as means in relation to the whole trial period and to live weight classes of the pigs. The concentrations of protein, urea, creatinine, LDL+VLDL cholesterol, GGTP and AP were significantly different between DS and DL both for whole trial period and for most of live weight classes. For urea acid, lactate, triglycerides, HDL cholesterol, ALAT and LDH breed differences were mainly small. The breed differences for the concentrations of glucose, total cholesterol, ASAT and CK were statistically significant in some live weight classes, only.
\end{abstract}

Key words: pig, German Saddle Back, German Landrace, blood traits, substrates, enzymes, weight

\title{
Zusammenfassung
}

Bei 28 Börgen der Rassen Deutsches Sattelschwein (DS) und 30 Deutsche Landrasse (DL) wurden während der Mast in vierzehntägigen Abständen Blutproben gewonnen. Dabei erfolgte die Blutprobenentnahme 24 Stunden nach Myostressinjektion aus der Vena cava cranialis. Aus dem Blutplasma wurden insgesamt 16 Blutmerkmale bestimmt. Zur Charakterisierung der Rassenunterschiede sind die Ergebnisse als Mittelwerte über den Gesamtversuchszeitraum bzw. in Abhängigkeit von Lebendgewichtsklassen der Tiere dargestellt worden. Bei den Blutmerkmalen Protein, Harnstoff, Kreatinin, LDL+VLDL-Cholesterin, GGTP und AP waren sowohl aus den Gesamtmittelwerten als auch aus den gewichtsabhängigen Mittelwerten statistisch gesicherte Unterschiede zwischen DS und DL nachweisbar. Für Harnsäure, Laktat, Triglyceride, HDL-Cholesterin, ALAT und LDH ergaben sich im Mittel keine bzw. gewichtsabhängig nur wenige signifikante Differenzen zwischen den beiden Rassen. Die Konzentrationen von Glukose, Gesamtcholesterin, ASAT und CK waren nur teilweise zwischen DS und DL signifikant verschieden. Schlüsselwörter: Schwein, Deutsches Sattelschwein, Deutsche Landrasse, Blutmetaboliten, Substrate, Enzyme,
Gewicht

\section{Einleitung}

Durch die Auswahl und Zuchtverwendung von Tieren mit hohen Leistungen konnten in der Tierzucht erhebliche Fortschritte erreicht werden. Die gesteigerte Leistungsfähigkeit moderner Schweinerassen gegenüber herkömmlichen Rassen begründet sich bei gleichen Umweltbedingungen vor allem in unterschiedlichen speziellen Leistungen 
der Tiere. Die differenzierte genetische Veranlagung induziert direkt oder indirekt die unterschiedliche Entwicklung und Leistungsausprägung des Organismus und damit die in den Geweben, Organen und im Blut ablaufenden Stoffwechselprozesse sowie ihre gegenseitige Beeinflussung und Regulierung. Im Vergleich zur Deutschen Landrasse stellt das Deutsche Sattelschwein einen sehr unterschiedlichen Typ dar, über deren Herkunft und differenzierte Mast- und Schlachtkörperqualität KUHN et al. (1997, 1998) berichteten.

Im folgenden Beitrag werden am gleichen Tiermaterial einzelne Blutkennwerte analysiert, um zu prüfen, inwieweit zwischen beiden Rassen unterschiedliche Konzentrationen für das jeweilige Merkmal vorliegen und welchen Einfluß die Gewichtsentwicklung der Tiere bei Blutentnahme auf die Merkmalsausprägung ausübt.

\section{Material und Methode}

Wie bei KUHN et al. $(1997,1998)$ dargestellt, wurden die zur Untersuchung verwendeten Tiere der Rassen Deutsches Sattelschwein (DS) und Deutsche Landrasse (DL) bei einem Lebendgewicht von durchschnittlich 28 bis $110 \mathrm{~kg}$ einer Mastleistungsprüfung mit einheitlicher Futtermischung (12,6 MJ umsetzbarer Energie, 17 \% Rohprotein, 3,3\% Rohfett und 5,6 \% Rohfaser) unterzogen. Anschließend wurden die Tiere geschlachtet und ihre Schlachtkörperqualität und Fleischbeschaffenheit bestimmt.

Während der Mastperiode wurden bei 28 Börgen der Rasse DS und bei 30 Börgen der Rasse DL, ab Versuchsbeginn bis zur Schlachtung, in vierzehntägigen Abständen Blutproben gewonnen.

Die Blutentnahme am Einzeltier erfolgte zeitgleich 24 Stunden nach einer standardisierten Belastung (Myostressinjektion) zur gleichen Tageszeit ( $8^{\circ \circ} \mathrm{Uhr}$ ) bei genüchterten Tieren durch Punktion aus der Vena cava cranialis. Das heparinisierte Blut wurde zentrifugiert, das Plasma abgefüllt und bis zur labortechnischen Bearbeitung eingefroren.

Aus dem Plasma sind insgesamt 16 Substrate und Enzyme photometrisch (Photometer LP 400) mit Hilfe standardisierter Testkits bestimmt worden (Tab. 1). Die Aktivität der Creatinkinase wurde in Anlehnung an BICKHARDT und RICHTER (1980) als Logarithmuswert angegeben, um bei diesem Merkmal einer Normalverteilung nahezukommen. Inwieweit wiederholte Myostressinjektionen bei den Tieren „Gewöhnungseffekte" hervorrufen, kann hier nicht beurteilt werden.

Für die Substratgehalte und Enzymkonzentrationen wurde eine Sortierung nach der Gewichtsentwicklung der Tiere in 10-kg-Stufen vorgenommen. Die Zuordnung der Blutproben zu den jeweiligen Gewichtsklassen der Tiere ergibt die in Tabelle 2 dargestellte Verteilung der Proben.

Die Signifikanz der Mittelwert-Differenzen wurde mit Hilfe des t-Tests geprüft, wobei eine Irrtumswahrscheinlichkeit von $P \leq 0,05$ bzw. $\leq 0,10$ zugrundegelegt wurde. Signifikant unterschiedliche Mittelwerte wurden mit den Buchstaben a und b bzw. mit + gekennzeichnet. 
Tabelle 1

Untersuchte Stoffwechselparameter des Blutes (Investigated metabolic traits in blood of pigs)

\begin{tabular}{|c|c|c|c|}
\hline Merkmal & Kurzbezeichnung & Maßeinheit & Bestimmungsmethode \\
\hline Gesamtprotein & Prot. & $\mathrm{g} / \mathrm{dl}$ & Biuret - Methode \\
\hline Harnstoff & Hst. & $\mathrm{mg} / \mathrm{dl}$ & vollenzym. UV-Test, kinetisch \\
\hline Harnsäure & Hsr. & $\mathrm{mg} / \mathrm{dl}$ & Trinder-Methode, enzym. Farbtest \\
\hline Glukose & Gluc. & $\mathrm{mg} / \mathrm{dl}$ & GOD-PAP-Methode \\
\hline Laktat & Lac. & $\mathrm{mmol} / 1$ & LOX-PAP-Methode \\
\hline Kreatinin & Krea. & $\mathrm{mg} / \mathrm{dl}$ & Jaffe - Reaktion \\
\hline Gesamtcholesterin & Chol. & $\mathrm{mg} / \mathrm{dl}$ & CHOD-PAP-Meth., enzym. Farbtest \\
\hline $\begin{array}{l}\text { High density lipoproteins- } \\
\text { cholesterol }\end{array}$ & HDL-Chol. & $\mathrm{mg} / \mathrm{dl}$ & mit spez. Fällungsreaktion \\
\hline $\begin{array}{l}\text { Low and very low density } \\
\text { lipoproteins-cholesterol }\end{array}$ & LDL + VLDL-Chol. & $\mathrm{mg} / \mathrm{dl}$ & mit spez. Fällungsreaktion \\
\hline Triglyceride & Trig. & $\mathrm{mg} / \mathrm{dl}$ & GPO-PAP-Meth., enzym. Farbtest \\
\hline Aspartat-Amino-transferase & ASAT (GOT) & $\mathrm{U} / \mathrm{l}$ & $\begin{array}{l}\text { optim. Standardmethode, DGKC, } \\
\text { Meßtemp. } 37^{\circ} \mathrm{C}\end{array}$ \\
\hline Alanin-Aminotransferase & $\operatorname{ALAT}(\mathrm{GPT})$ & $\mathrm{U} / 1$ & optim. Standardmethode , \\
\hline Laktatdehydrogenase & $\mathrm{LDH}$ & $\mathrm{U} / 1$ & optim. Standardmethode , \\
\hline Creatinkinase- $\mathrm{lg}$ & CK-lg & $\mathrm{U} / \mathrm{l}-\mathrm{lg}$ & $\begin{array}{l}\text { optim. Standardmethode , } \\
\text { NAC - Akt. }\end{array}$ \\
\hline $\begin{array}{l}\text { Gamma-Glutamyl- } \\
\text { Transpeptidase }\end{array}$ & GGTP & $\mathrm{U} / \mathrm{I}$ & Farbtest nach Szasz \\
\hline $\begin{array}{l}\text { Alkalische } \\
\text { Phosphatase }\end{array}$ & AP & $\mathrm{U} / 1$ & optim. Standardmethode , \\
\hline
\end{tabular}

\section{Tabelle 2}

Anzahl Blutproben in verschiedenen Lebendgewichtsklassen (Number of blood samples at different live weight classes of pigs)

\begin{tabular}{ccc}
\hline $\begin{array}{c}\text { Gewichtsklasse } \\
(\mathrm{kg})\end{array}$ & $\begin{array}{c}\text { Deutsches } \\
\text { Sattelschwein } \\
\mathbf{n}\end{array}$ & $\begin{array}{c}\text { Deutsche } \\
\text { Landrasse } \\
\mathrm{n}\end{array}$ \\
\hline$<29,9$ & 11 & 5 \\
$30-39,9$ & 18 & 16 \\
$40-49,9$ & 19 & 8 \\
$50-59,9$ & 16 & 11 \\
$60-69,9$ & 17 & 12 \\
$70-79,9$ & 12 & 12 \\
$80-89,9$ & 19 & 10 \\
$90-99,9$ & 21 & 8 \\
$>100$ & 21 & 10 \\
\hline
\end{tabular}

\section{Ergebnisse}

Im Ergebnis des Vergleichs zur Schlachtkörperqualität von Deutschen Sattelschweinen und Landrasseschweinen zeigen sich erhebliche Unterschiede zwischen den Rassen 
(Tab. 3). Bei Muskelfleischanteilen von 52,9 \% (DL) und 40,8\% (DS) wurden Fettgewebeanteile (ohne Innenfett) von $28,2 \%$ bei DL bzw. $40,9 \%$ bei DS ermittelt. Ebenso lag der Auflagefettanteil bei DS um 9,3\% höher als bei DL. Auch der Anteil des inter- und intramuskulären Fettes war bei den Sattelschweinen größer als bei den Landrassetieren. Diese extremen Unterschiede scheinen auch verschiedene Stoffwechselabläufe zu beeinflussen, die im folgenden anhand von Blutmerkmalen analysiert werden sollen.

Tabelle 3

Gewebeanteile am Schlachtkörper von DL und DS (Carcass tissue percentage of DL and DS)

\begin{tabular}{|c|c|c|c|c|c|}
\hline \multirow[t]{2}{*}{ Rasse } & & \multicolumn{2}{|c|}{ DL } & \multicolumn{2}{|c|}{ DS } \\
\hline & & $\overline{\mathbf{x}}$ & s & $\overline{\mathrm{x}}$ & s \\
\hline Muskelfleisch & $\%$ & 52,9 & 0,68 & 40,8 & 0,67 \\
\hline $\begin{array}{l}\text { Fettgewebe } \\
\text { (ohne Innenfett) }\end{array}$ & $\%$ & 28,2 & 0,78 & 40,9 & 0,77 \\
\hline Auflagefett & $\%$ & 19,5 & 0,63 & 28,8 & 0,62 \\
\hline Intermuskuläres Fett & $\%$ & 8,6 & 0,21 & 12,2 & 0,21 \\
\hline Intramuskuläres Fett & $\%$ & 1,3 & 0,13 & 2,9 & 0,13 \\
\hline
\end{tabular}

Tabelle 4

Mittelwerte und Standardabweichungen der untersuchten Blutmerkmale für die Rassen DS und DL (Means and standard deviations for the tested blood traits in DS and DL)

\begin{tabular}{|c|c|c|c|c|c|}
\hline \multirow[t]{2}{*}{ Blutmerkmal } & \multirow[t]{2}{*}{ Maßeinheit } & \multicolumn{2}{|c|}{$\begin{array}{l}\text { DS } \\
134)\end{array}$} & \multicolumn{2}{|c|}{$\begin{array}{r}\text { DL } \\
(n=93)\end{array}$} \\
\hline & & $\overline{\mathbf{x}}$ & s & $\overline{\mathrm{x}}$ & $s$ \\
\hline Protein & $\mathrm{g} / \mathrm{dl}$ & $6,17^{2}$ & 0,82 & $5,75^{b}$ & 0,66 \\
\hline Harnstoff & $\mathrm{mg} / \mathrm{dl}$ & $17,4^{a}$ & 10,7 & $12,3^{b}$ & 7,74 \\
\hline Harnsäure & $\mathrm{mg} / \mathrm{dl}$ & 0,31 & 0,38 & 0,27 & 0,23 \\
\hline Glukose & $\mathrm{mg} / \mathrm{dl}$ & 91,2 & 14,0 & 93,2 & 12,2 \\
\hline Laktat & $\mathrm{mmol} / \mathrm{l}$ & 7,50 & 3,31 & 8,12 & 4,74 \\
\hline Kreatinin & $\mathrm{mg} / \mathrm{dl}$ & $0,91^{a}$ & 0,15 & $1,08^{b}$ & 0,18 \\
\hline Gesamt-Chol. & $\mathrm{mg} / \mathrm{dl}$ & $76,0^{\mathrm{a}}$ & 13,3 & $86,1^{\mathrm{b}}$ & 13,7 \\
\hline HDL-Chol. & $\mathrm{mg} / \mathrm{dl}$ & 32,8 & 9,4 & 32,3 & 8,1 \\
\hline LDL+VLDL-Chol. & $\mathrm{mg} / \mathrm{dl}$ & $43,2^{n}$ & 10,6 & $53,8^{\mathrm{b}}$ & 12,0 \\
\hline Triglyceride & $\mathrm{mg} / \mathrm{dl}$ & 20,8 & 9,34 & 23,2 & 11,5 \\
\hline ASAT & $\mathrm{U} / 1$ & $99,3^{2}$ & 61,3 & $131,1^{\mathrm{b}}$ & 102,5 \\
\hline ALAT & $\mathrm{U} / 1$ & 75,3 & 29,6 & 85,7 & 45,7 \\
\hline $\mathrm{LDH}$ & $\mathrm{U} / 1$ & 816 & 375 & 836 & 379 \\
\hline CK-lg & $\mathrm{U} / 1$ & $2,73^{\mathrm{x}}$ & 0,46 & $2,99^{b}$ & 0,37 \\
\hline GGTP & $\mathrm{U} / \mathrm{I}$ & $19,2^{x}$ & 13,1 & 27,1 & 10,5 \\
\hline AP & $\mathrm{U} / \mathrm{I}$ & $196^{a}$ & 61,3 & $257^{b}$ & 64,5 \\
\hline
\end{tabular}

Signifikante Differenzen zwischen DS und DL (PS 0,05): a,b

Die untersuchten Blutkennwerte von Börgen der Rassen DS und DL unterscheiden sich bei Zusammenfassung aller Gewichtsklassen teilweise signifikant voneinander (Tab. 4). So ergeben sich bei DS-Tieren höhere Protein- und Harnstoffgehalte im Blut als bei DL-Tieren, wobei bei beiden Merkmalen im Vergleich zu Referenzwerten (SCHMIDL und FORTNER, 1985) niedrigere Konzentrationen gefunden wurden. Bei den DS-Tieren ist im Vergleich zu den DL eine Erhöhung der Kreatininkonzentration im Blut nachzuweisen. Dagegen sind bei DL-Tieren im Mittel der Blutproben signifi- 
kant höhere Gesamt- und LDL+VLDL-Cholesterinkonzentrationen als bei den DS-Tieren ermittelt worden.

Die folgenden graphischen Darstellungen zeigen Ergebnisse für die einzelnen Blutparameter. Dabei werden die untersuchten Aktivitäts- bzw. Konzentrationswerte als Mittelwerte in den neun ausgewiesenen Gewichtsklassen im Vergleich der beiden Rassen DS und DL dargestellt.

Die Untersuchungsergebnisse zur Konzentration der Substrate im Blutplasma sind aus Abbildung 1 ersichtlich. Übereinstimmend ergeben sich zu allen Untersuchungszeitpunkten bei DS-Tieren höhere Proteinplasmawerte als bei DL-Tieren.
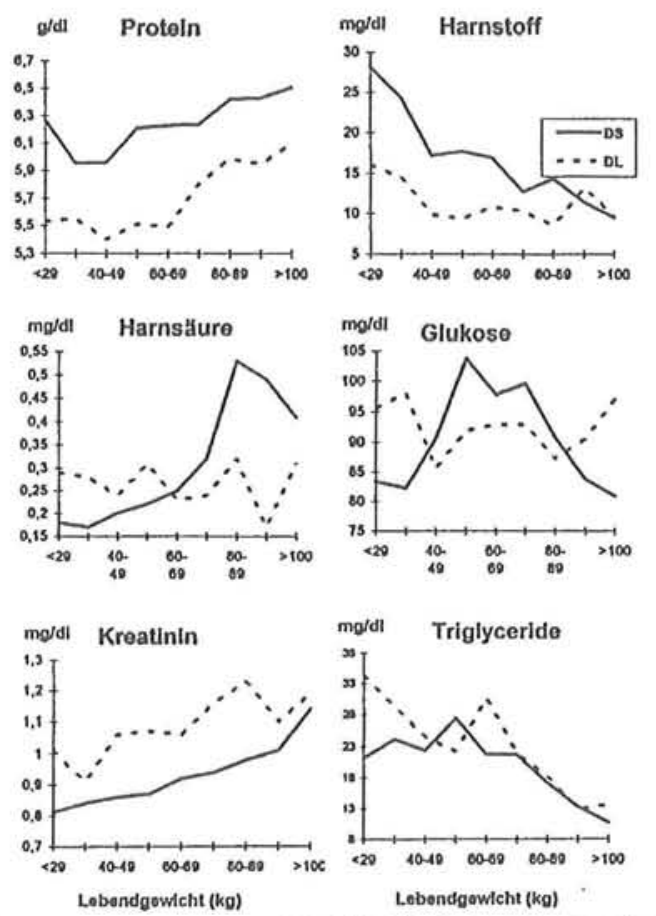

Abb. 1: Substrate im Blutplasma in Abhängigkeit
vom Lebendgewicht der Schweine (Blood
substrates in pigs at different live weights)

Ausdruck der sich aus dem Proteinüberschuß ergebenden Ausscheidungsrate ist unter anderem die Harnstoffkonzentration des Blutes, wenn sie auch im Vergleich zu Referenzwerten deren Umfang nicht dimensioniert. Der Harnstoffgehalt ist bei den DSSchweinen bis auf die Endmastphase stets höher als bei den DL-Schweinen. Zu Beginn der Untersuchungen haben DS mit $28 \mathrm{mg} / \mathrm{dl}$ fast eine doppelt so hohe Konzentration im Blut als Tiere der Rasse DL. Im Laufe der Entwicklung der Schweine verringert sich dieser Unterschied immer mehr und ist bei $90 \mathrm{~kg}$ Gewicht praktisch nicht mehr vorhanden. Dabei ist bei DS-Tieren mit steigendem Lebendgewicht ein deutlicher Abfall der Harnstoffkonzentration im Blutplasma zu registrieren.

Es ist ersichtlich, daß sich bei der Harnsäurekonzentration des Blutes unterschiedliche Verläufe zwischen den untersuchten Rassen ergeben. Während bei den DL-Schweinen 
in allen Gewichtsklassen relativ konstante Blutwerte zu finden sind, erhöht sich die Konzentration der Harnsäure im Blut mit zunehmendem Gewicht der DS-Tiere.

Die Mittelwerte für Glukose zu einzelnen Blutentnahmezeitpunkten variieren im Bereich von 80 bis $105 \mathrm{mg} / \mathrm{dl}$. Bei den DS-Tieren sind bei Körpergewichten von 50 bis $80 \mathrm{~kg}$ höhere Glukosekonzentrationen ersichtlich als zum Beginn und Ende der Mastprüfperiode. Die Werte zeigen dagegen bei DL ein annähernd entgegengesetztes Bild. Zum Anfang und zum Ende des Mastzeitraums wurden höhere Gehaltswerte ermittelt als bei einem Gewicht von 50 bis $80 \mathrm{~kg}$. Die im Vergleich zu Referenzwerten relativ hohen Glukoseblutwerte bei beiden Rassen können durch die Myostressinjektion mit induziert sein.

Kreatinin steht mit der Muskelmasse des Körpers in positiver Beziehung. Bei beiden untersuchten Rassen ist mit steigenden Körpergewichten eine Zunahme der Kreatininkonzentration im Plasma ersichtlich. Beim DL wurden durchschnittlich um 10 bis $20 \%$ höhere Kreatininwerte als bei DS ermittelt. Dieser Befund kann mit dem höheren Muskelfleischanteil der DL-Tiere in Verbindung stehen. Die Triglyceridkonzentration des Blutplasmas unterscheidet sich zwischen den Rassen im Verlauf der untersuchten Wachstumsphase nicht wesentlich. Nur bei 30 bis $40 \mathrm{~kg}$ Körpergewicht sind bei DL höhere Konzentrationen als bei DS zu ersehen. Insgesamt verringern sich mit steigenden Körpergewichten bei beiden Rassen die Triglyceridwerte im Blut. Da Cholesterin im Blut sehr differenziert transportiert wird, wurden neben dem Gesamtcholesterin auch die an „high density lipoproteins-Cholesterin“(HDL-Cholesterin) bzw. an „low density lipoproteins plus very low density lipoproteins-Cholesterin"(LDL+VLDLCholesterin) gebundenen Cholesterin-Untergruppen bestimmt und in Abbildung 2 in Abhängigkeit vom Gewicht der Tiere für beide untersuchten Rassen dargestellt.
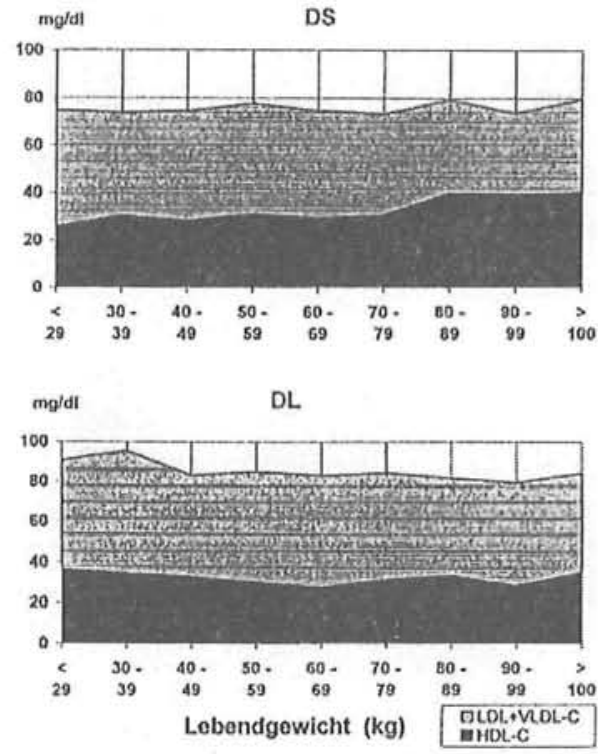

Abb. 2: Konzentration von Gesamtcholesterin, HDL- und LDL+VLDL- Cholesterin im Blut in Abhängigkeit vom Lebendgewicht der Schweine (Blood concentration of total cholesterol, HDL- and LDL+VLDL cholesterol in pigs at different live weights) 
Bei DS sind in allen Lebendgewichtsabschnitten etwa gleich hohe Gesamtcholesterinkonzentrationen von $76 \mathrm{mg} / \mathrm{dl}$ gefunden worden. Bei den DL ergeben sich mit steigenden Körpergewichten leicht sinkende Konzentrationen, welche sich von $93 \mathrm{mg} / \mathrm{dl}$ bei $30 \mathrm{~kg}$ Gewicht auf $85 \mathrm{mg} / \mathrm{dl}$ bei $100 \mathrm{~kg}$ Gewicht verringern. DL-Schweine haben einen LDL+VLDL-Cholesteringehalt, der im Mittel gleichbleibend bei $54 \mathrm{mg} / \mathrm{dl}$ liegt, während die analogen Werte bei den DS ca. $43 \mathrm{mg} / \mathrm{dl}$ betragen und eine sich mit steigendem Gewicht verringernde Konzentration aufweisen. Im Rassenvergleich ergibt sich beim Merkmal HDL-Cholesterin, daß die DL-Tiere einen während der Mastperiode etwa gleichbleibend hohen Gehalt im Plasma haben, während HDL-Cholesterin bei DS mit zunehmendem Gewicht ansteigt. Damit differieren die HDL-Cholesterin-Gehalte bei beiden Rassen besonders bei niedrigen und hohen Gewichten.
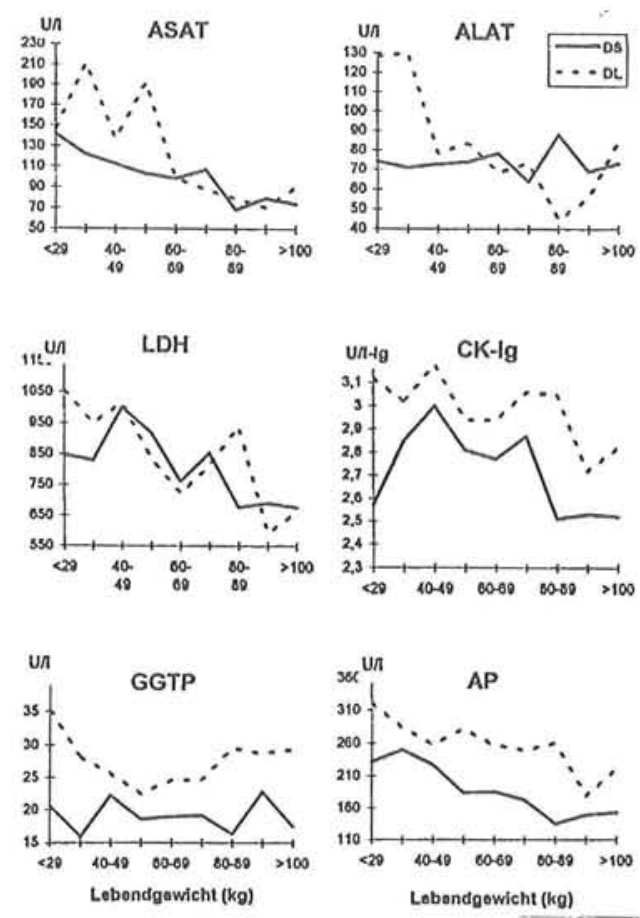

Abb. 3: Enzymaktivitäten im Blutplasma in Abhängigkeit von Lebendgewicht der Schweine (Enzyme activity in blood plasma in pigs at different live weights)

Die Enzymaktivitäten von Aspartat-Aminotransferase und Alanin-Aminotransferase (Abb. 3) lassen keine eindeutigen Differenzierungen zwischen DL- und DS-Tieren erkennen. Charakteristisch ist, daß bei den jungen Tieren Unterschiede in den Enzymaktivitäten bestehen, die sich ab 50 bis $60 \mathrm{~kg}$ Lebendgewicht nicht mehr abzeichnen. In der Tendenz sinken die Enzymaktivitäten mit zunehmenden Gewichten der Tiere ab. Sie sind jedoch insgesamt als hoch zu bewerten, wenn sie mit Referenzwerten verglichen werden.

Mit steigendem Gewicht der Tiere verringert sich die Laktatdehydrogenase-(LDH)Aktivität im Blutplasma bei beiden Rassen. Es ergeben sich keine rassenspezifischen Verläufe für dieses Enzym. 
Das Enzym Creatinkinase wird nach Myostressinjektion zur Bewertung der Stressanfälligkeit der Tiere herangezogen und ist damit auch höher als bei den Referenzwerten angegeben. Bei Landrasseschweinen variieren die mittleren CK-lg-Aktivitäten in Abhängigkeit vom Gewicht der Tiere im Bereich von 3,0 bis 3,2 U/1. Bei Sattelschweinen liegen die analogen Aktivitäten mit 2,8 bis 2,9 U/l geringfügig, aber einheitlich, tiefer. Während bei beiden Rassen Tiere mit geringer Lebendmasse mittlere CK-lg-Aktivitäten um 2,8 bis 3,1 U/l aufweisen, verringern sich bei Tieren mit über $80 \mathrm{~kg}$ Lebendgewicht bei beiden Rassen die Enzymaktivitäten.

Besonders charakteristisch für die Rassenunterschiede zwischen DL und DS scheinen die Enzyme Gamma-Glutamyl-Transpeptidase und Alkalische Phosphatase zu sein.

Tabelle 5

Signifikanz der Mittelwertdifferenzen zwischen den Rassen DS und DL für die untersuchten Blutmerkmale in Abhängigkeit vom Lebendgewicht der Tiere (Significance of mean differences for investigated blood traits in relation to live weight of DS- and DL-pig)

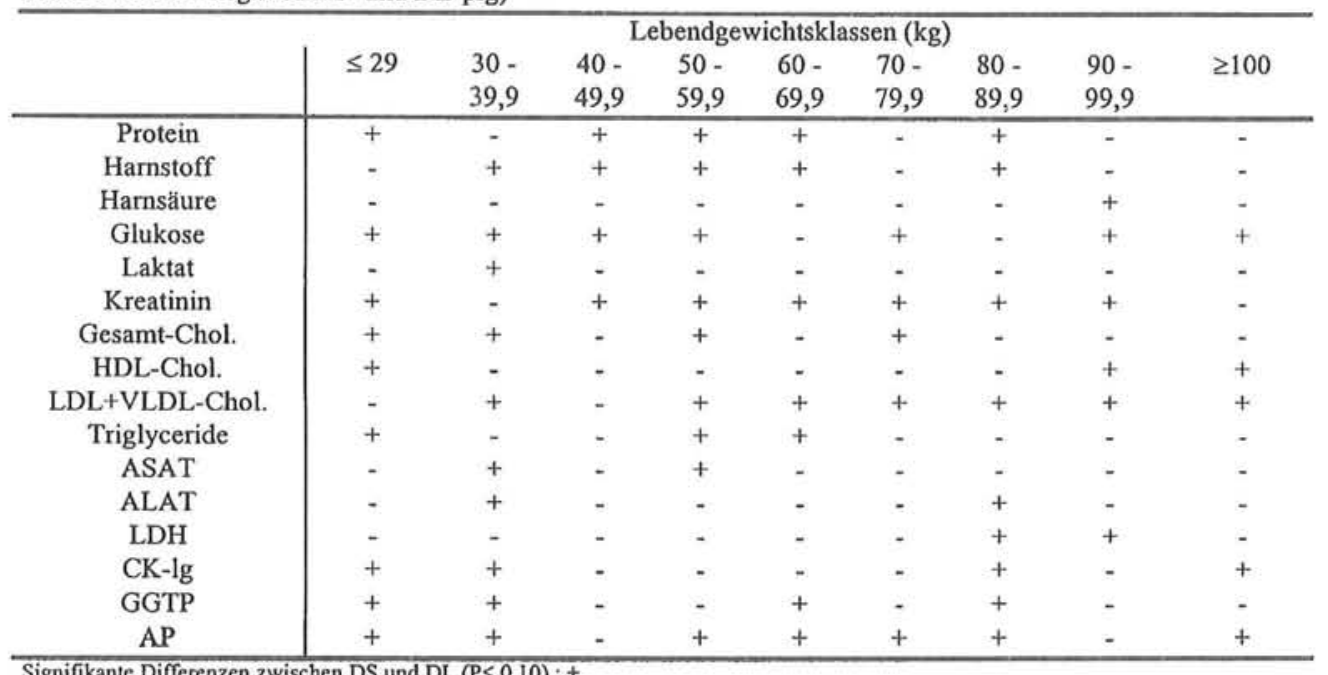

Signifikante Differenzen zwischen DS und DL (PS 0,10) : +

In Tabelle 5 sind Rassenunterschiede bei den Blutmerkmalen in Abhängigkeit vom Gewicht der Tiere zusammengefaßt worden. Dabei wurde ein Signifikanzwert von $\mathrm{P} \leq$ 0,10 zur Sicherung der Unterschiede zwischen den Rassen verwendet. Die Resultate zeigen, daß bei allen Blutmerkmalen gewichtsabhängige, signifikante Differenzen vorhanden sind. Dabei reicht das Spektrum der Unterschiede zwischen den Rassen von nur wenigen Gewichtsklassen je Merkmal (Harnsäure, Laktat, HDL-Chol., ASAT, ALAT, LDH) bis zu signifikanten Unterschieden bei den meisten Gewichtsklassen je Merkmal (Protein, Harnstoff, Glukose, Kreatinin, LDL+VLDL-Chol., GGTP, AP). Die ermittelten Unterschiede stimmen im wesentlichen mit den Ergebnissen aus Tabelle 4 überein, in der die Mittelwerte der Rassen über den gesamten Versuchszeitraum für die Einzelmerkmale verwendet wurden.

5. Diskussion

Als Übersicht zu den vielfältigen Wirkungen der untersuchten Substrate und Enzyme 
des Blutes wird auf Arbeiten und Zusammenstellungen von LENGERKEN und ALBRECHT (1977), SCHENK und KOLB (1982), LÖFFLER et al. (1990), FALKENBERG et al. (1996) sowie MICKLICH (1997) verwiesen. Die verwendeten Referenzwerte beziehen sich überwiegend auf Angaben von SCHMIDL und FORTNER (1985), da sie nach der methodischen Erfassung am besten mit den hier genutzten Untersuchungsmethoden übereinstimmen.

Insgesamt stellt das Blut ein offenes System dar, das mit allen Zellen und Organen des Körpers in Verbindung und im Austausch steht. Aus diesem Grunde ist die Homöostase des Blutes als Resultat von Stoffwechsel- und Umwelt-Organismus-Interaktionen anzusehen, die in charakteristischer Weise auch auf Unterschiede zwischen Individuen oder genetisch differenzierten Gruppen von Individuen hinweisen (ELSTNER, 1990; KLEBER und SCHLEE, 1991). Unter diesen Aspekten wurde versucht, über Stoffwechselparameter Unterschiede zwischen den beiden untersuchten Schweinerassen DS und DL nachzuweisen.

Die Blutmerkmale wurden, wie bereits im Methodenteil dargelegt, nach Myostresseinwirkung untersucht. Dadurch kann eine Standardisierung der Stoffwechselwirkungen erreicht werden, die eine bessere Vergleichbarkeit der Blutwerte ermöglichen. In diesem Zusammenhang müssen auch die Auslenkungen in der Konzentration einiger untersuchter Merkmale, wie sie an den Aktivitäten von CK, ASAT und ALAT deutlich werden, gesehen werden. Hohe ASAT- und LDH-Aktivitäten bei DL weisen gegenüber DS auf eine Belastung von Herz, Leber und Skelettmuskulatur hin. Sie unterstreichen ebenso wie signifikant erhöhte GGTP-, AP- und CK-Werte die unterschiedliche Stoffwechselaktivität dieser DL-Tiere im Vergleich zu den Sattelschweinen.

Bei gleichem Nährstoffangebot scheinen die fleischreicheren Landrassetiere das mit dem Futter aufgenommene Eiweiß besser auszunutzen als die fetteren Sattelschweine. In der als besonders intensiv zu bezeichnenden Wachstumsphase von etwa 40 bis $70 \mathrm{~kg}$ Lebendgewicht deuten niedrigere Proteinkonzentrationen bei DL auf eine besonders gute Eiweißverwertung hin. Bei den DS-Schweinen beschränkt sich die minimale Proteinkonzentration auf den Gewichtsabschnitt zwischen 30 und $50 \mathrm{~kg}$. In den höheren Gewichtsabschnitten nimmt die Proteinkonzentration im Blut bei beiden Rassen $\mathrm{zu}$, wenn auch mit unterschiedlichem Niveau und weist auf eine sich möglicherweise verschlechternde, nach Rassen differenzierte Proteinverwertung hin. Wie bereits angeführt, sind Normalwerte dieses Merkmals noch höher (SCHMIDL und FORTNER, 1985), als bei den untersuchten Tieren ermittelt.

Es zeigte sich, daß die untersuchten biochemischen Blutmerkmale in unterschiedlicher Weise geeignet sind, die Spezifik der in vorliegender Arbeit verglichenen Rassen darzustellen. Bei einem Teil der Merkmale, wie der Harnsäure, HDL-Chol., ALAT und LDH ergaben sich keine bzw. nur geringe Differenzen zwischen den Tieren der beiden Rassen. Bei den Merkmalen Protein, Harnstoff, Kreatinin, LDL+VLDL-Chol., GGTP und AP waren sowohl aus den Gesamtmittelwerten als auch aus den gewichtsabhängigen Darstellungen statistisch gesicherte Unterschiede zwischen DS und DL ersichtlich, die für eine genaue Rassendifferenzierung genutzt werden können. Da z. B. eine hohe GGTP-Aktivität eine hohe Aufnahmekapazität von Aminosäuren in die Zellen indu- 
ziert, sind daraus bei DL-Tieren gegenüber DS-Tieren wesentlich höhere Wachstumsleistungen abzuleiten. Die AP-Konzentration bei DL-Tieren verweist auf die im gesamten Untersuchungszeitraum höhere Skelettbildung bei diesen Tieren gegenüber DS-Tieren und steht in Übereinstimmung mit dem höheren Wachstum der DLSchweine. Eine hohe Variabilität der Merkmale war innerhalb der Gewichtsklassen zu beobachten. Es ist zu erwarten, daß Mittelwerte aus mehreren Messungen je Merkmal innerhalb gleicher Gewichtsklassen oder im Verlauf des Wachstums sich möglicherweise besser zur Bewertung der Rassen als einzelne Blutwerte eignen (FALKENBERG et al., 1996). Ein relativ einheitlicher Gewichtsabschnitt ist jedoch zu bevorzugen.

Bei einer Reihe von Merkmalen ist auffällig, daß speziell in den Gewichtsbereichen von 30 bis $50 \mathrm{~kg}$ größere Unterschiede zwischen den beiden Rassen bestehen als bei höheren Gewichten. Das ist unter anderem an den Merkmalen Harnstoff, Laktat, Gesamtcholesterin und ASAT ersichtlich. In diesem Entwicklungsstadium scheinen die Stoffwechselunterschiede zwischen den DL- und DS-Tieren häufig stärker ausgeprägt zu sein als zu späteren Zeitpunkten. Auch zeigt sich bei vielen Merkmalen beider Rassen ein Abfall der untersuchten Blutkonzentrationswerte mit steigenden Lebendgewichten. Zusammenfassend scheint zwischen den in Tabelle 3 dargestellten und ausführlich bei KUHN et al. $(1997,1998)$ beschriebenen Unterschieden hinsichtlich spezieller Merkmale und den hier untersuchten Blutkennwerten von Schweinen beider Rassen eine mehr oder weniger ausgeprägte Wechselwirkung zu bestehen. In vielen Fällen weisen signifikante Unterschiede zwischen den untersuchten Parametern auf einen rassenspezifischen Metabolismus hin.

\section{Literatur}

BICKHARDT, K.; RICHTER, L.: Methodische Aspekte des CK-Tests beim Schwein. Dt. tierärztl. Wschr. Hannover 87 (1980), 296-298 ELSTNER, E. F.: Der Sauerstoff. Wissenschaftsverl., Mannheim, Wien, Zürich, 1990

FALKENBERG, H.; MICKLICH, D; MATTHES, H.-D.; MÖRING, H.: Blutkennwerte verschiedener Schweinerassen unter dem Einfluß von Stall- und Freilandhaltung. Arch Tierz., Dummerstorf 39 (1996), 153-168

KLEBER, H.-P.; SCHLEE, D.: Biochemie. Teil 1: Allgemeine und funktionelle Biochemie. G. Fischer Verlag Jena, 1991

KUHN, G.; HARTUNG, M.; FALKENBERG, H.; NUURNBERG, G.; LANGHAMMER, M.; SCHWERIN, M.; ENDER, K.:

Wachstum, Körperzusammensetzung und Fleischbeschaffenheit von im Fettansatz genetisch differenten Schweinen. Arch. Tierz., Dummerstorf 40 (1997), 345-355

KUHN, G.; NƯRNBERG, K.; FIEDLER, I.; FALKENBERG, H.; NƯRNBERG, G.; ENDER, K.: Körperzusammensetzung und Muskelstruktur von genetisch differenten Schweinen in Abhängigkeit vom MHS-Status. Arch. Tierz., Dummerstorf 41 (1998), 589-596

LENGERKEN, G. von; ALBRECHT, V.:

Eignung biochemischer Kennwerte und ausgewählter Regulationssysteme für die Früherkennung einer Prädisposition zur Ausbildung von Fleischqualitătsmängeln beim Schwein. Habilschr., Univ. Leipzig, 1977

LÖFFLER, G.; PETRIDES, E.; WEISS, L.:

Physiologische Chemie. Springer-Verlag, Berlin, 1990, 4. Aufl. 
MICKLICH, D.:

Die Wirkung der Weidehaltung verschiedener Schweinerassen auf die natürliche Sukzession von Flußauengrïnland und den physiologischen Zustand der Sauen. Univ. Rostock, Diss., 1997

SCHENK, M.; KOLB, E.:

Grundriß der physiologischen Chemie. G. Fischer Verl., Jena, 1982

SCHMIDL, M.; FORTNER, S.:

Veterinärmedizinische Laboruntersuchungen für die Diagnose und Verlaufskontrolle. Boehringer, Mannheim, 1985, 3. Aufl.

Eingegangen: 30.04 .1998

Akzeptiert: 14.01 .1999

Anschriften der Verfasser

Dr. habil. HEINZ FALKENBERG

Institut für Angewandte Agrarökologie

Justus-von-Liebig-Weg 8

D-18059 Rostock

Dr. GERDA KUHN, Dr. MARGITTA HARTUNG, Dr. MARTINA LANGHAMMER

Forschungsinstitut für die Biologie landwirtschaftlicher Nutztiere

Wilhelm-Stahl-Allee 2

D-18196 Dummerstorf

Dr. CAROLA WOLF

Landesveterinär- und Lebensmitteluntersuchungsamt Rostock

Thierfelder Straße 8

D-18059 Rostock 


\title{
Buchbesprechung
}

\author{
Grundkurs in klassischer Homöopathie für Tierärzte
}

ANDREAS SCHMIDT

2. Auflage, 240 Seiten, zahlreiche Tabellen und Übersichten, Sonntag Verlag Stuttgart, 1998, ISBN 3-87758-163-3, 68,-- DM, 496,-- ÖS, 62,-- SFR

Seit ihren Anfängen hat die Homöopathie immer wieder Eingang in die Tiermedizin gefunden. Die Gründe in neuerer Zeit liegen wohl darin, daß Nutztierhalter vermehrt "nicht chemische" Therapien fordern, auch um Absetzfristen nach Chemotherapien zu umgehen. Erwähnt sei auch die Gruppe der sog. Biolandwirte, die homöopathische Behandlungen als Ergänzung, nicht als Ersatz schulmedizinischer Therapien bevorzugen. Andererseits nimmt die Zahl der Tierärzte, die neben der offiziellen Lehrmeinung auch homöopathische Überlegungen in ihre Therapien einbringen ständig zu, was z.T. darin begründet sein mag, daß bei bestimmten Krankheiten homöopathische Mittel nicht nur auch, sondern sogar besser wirken können. Übersieht man die homöopathische Literatur so ist festzustellen, daß in jüngerer Zeit aus dem Kreis der Tierärzte eine recht umfängliche Literatur über Alternativen zu konventionellen Behandlungsmethoden vorliegt, wobei es sich meist um die Mitteilungen persönlicher Erfahrungen handelt. Doch fehlt so etwas wie ein Grundkurs für Tierärzte und interessierte Landwirte. Diesem Bedürfnis wollten der Herausgeber und seine Koautoren mit vorliegendem Buch entsprechen. Sie verstehen diesen Grundkurs als Hilfe für den Einstieg in diese Behandlungsmethoden im Sinne von Ergänzung für schulmedizinische Therapien.

Die nach nur vier Jahren nach dem Erscheinen der ersten Auflage vorliegende, überarbeitete und um wichtige Abschnitte erweiterte Neuauflage, hält sich in ihrer Gliederung an die erste Auflage. Nach den beiden ersten Abschnitten zur heutigen Stellung und Geschichte der Homöopatie, die interessant und wertend diskutiert wird, folgen acht Hauptkapitel. Sie beinhalten u.a. die Grundlagen, Arzneimittellehre und Repertorien sowie die Arzneimittel der Homöopathie. Es folgen die Fallaufnahme, Wahl der geeigneten Arzneimittel, die Behandlung akuter und chronischer Krankheiten, Konsultation und die weiteren Behandlungen sowie Behandlungshinweise wie zur Notfalltherapie, zu Verhaltensstörungen u.a. Es wird gezeigt, wie man zu den klinischen Ergebnissen gelangt und wie methodisch richtig gearbeitet wird. Ein Anhang gibt Hinweise zu Fort- und Weiterbildungsmöglichkeiten in der Veterinärhomöopathie. Neben dem jedem Kapitel angefügten Literaturverzeichnis, wurde der Abschnitt „Literatur der Homöopathie“" gründlich überarbeitet.

Obwohl 11 Autoren beteiligt sind, zeichnet sich das Buch durch einheitliche Vorgehensweise, eine gute Didaktik im Sinne eines Grundkurses aus und ist reich gegliedert. Seine Übersichtlichkeit wird durch zahlreiche drucktechnische Besonderheiten wirkungsvoll unterstützt.

Das Buch macht deutlich, daß Homöopathie grundsätzlich eine Regulationstherapie ist, welche fehlgewordene Anpassungsfähigkeit des Organismus im Sinne einer Selbstheilung korrigiert bzw. fördert. Sie ist für den Anwender an bestimmte Voraussetzungen gebunden. So erfordert ihre Anwendung das tierärztliche Wissen, medizinisches Können, Beobachtungsgabe sowie die Fähigkeit der Deduktion von Befunden und deren Interpretation. Neben Tierärzten und Tierhaltern wendet sich dieses Buch vor allem an Tierärzte, die bereit sind, die eigenen medizinischen Strategien neu zu überdenken. Das Buch ist einem weiten Leserkreis zu empfehlen. 